\title{
Objeto digital em enfermagem neonatal: impacto na aprendizagem de estudantes
}

\author{
Digital object in neonatal nursing: impact on student learning
}

Natália Del'Angelo Aredes ${ }^{1}$, Fernanda dos Santos Nogueira de Góes ${ }^{2}$, Marta Angélica lossi Silva ${ }^{3}$, Marlene Fagundes Carvalho Gonçalves ${ }^{4}$, Luciana Mara Monti Fonseca ${ }^{5}$

\footnotetext{
${ }^{1}$ Enfermeira, Mestre em Enfermagem em Saúde Pública. Discente do Programa de Pós-Graduação Enfermagem em Saúde Pública, nível Mestrado, da Escola de Enfermagem de Ribeirão Preto da Universidade de São Paulo (EERP/USP). Ribeirão Preto, SP, Brasil. E-mail: nataliadel.aredes@gmail.com.

${ }^{2}$ Enfermeira, Doutora em Enfermagem em Saúde Pública. Professor Doutor da EERP/USP. Ribeirão Preto, SP, Brasil. E-mail: fersngoes@eerp.usp.br.

${ }^{3}$ Enfermeira, Doutora em Enfermagem em Saúde Pública. Professor Associado da EERP/USP. Ribeirão Preto, SP, Brasil. E-mail: maiossi@eerp.usp.br.

${ }^{4}$ Pedagoga, Doutora em Educação. Professor Doutor, da EERP/USP. Ribeirão Preto, SP, Brasil. E-mail: mgoncalves@eerp.usp.br.

${ }^{5}$ Enfermeira, Doutora em Enfermagem em Saúde Pública. Professor Associado da EERP/USP. Ribeirão Preto, SP, Brasil. E-mail: lumonti@eerp.usp.br.
}

\section{RESUMO}

O estudo comparou a aprendizagem cognitiva de estudantes de enfermagem, sobre avaliação clínica do prematuro, dividindo-os entre os que utilizaram e que não utilizaram objeto digital de aprendizagem (ODA) como material de apoio. Além disso, avaliou o ODA na opinião dos estudantes. O quase-experimento foi conduzido no contexto da disciplina de saúde infantil de um curso de Enfermagem, em que participaram 22 estudantes divididos entre grupo controle ( $\mathrm{n}=10$ ) e grupo experimental $(n=12)$. Não houve diferença estatisticamente significativa entre os grupos, porém a comparação do desempenho intragrupos demonstrou diferença significativa em ambos, denotando os efeitos positivos da aprendizagem. Quanto à opinião dos estudantes, foram obtidas avaliações muito satisfatórias sobre o objeto digital e suas características de interação com o usuário. Apesar de não haver impacto significativo na comparação entre os grupos, a avaliação positiva de uso pelos estudantes reflete sua importância enquanto estratégia de inovação didática e motivação para os estudos.

Descritores: Educação em Enfermagem; Tecnologia Educacional; Enfermagem Neonatal.

\section{ABSTRACT}

The study compared the cognitive learning of nursing students about clinical evaluation of premature, dividing them among those who used and did not use the learning digital object (LDO) as support material. Besides, it assessed the students' opinion about the LDO. The quasi-experiment was conducted in the infant health discipline of a Nursing course, in which 22 students participated divided as control group $(n=10)$ and experimental group $(n=12)$. There was no significant statistical difference between groups, but the intra-group comparison for performance demonstrated significant difference in both, showing positive learning effects. Regarding students' opinion, very satisfying assessments were obtained about the digital object and its characteristics of interaction with the user. Although there was no significant impact between groups, the positive assessment from students' use reflects its importance as innovative didactic strategy and motivation for the students.

Descriptors: Education, Nursing; Educational Technology; Neonatal Nursing. 


\section{INTRODUÇÃO}

Os objetos digitais de aprendizagem (ODA), também conhecidos como objetos virtuais de aprendizagem, têm ganhado ênfase na estratégia pedagógica de curso superior, dadas suas vantagens de reutilização, interação motivadora por meio de animações ou simulações e aplicação versátil para ensino a distância ou presencial. Apesar disto, sua expansão na área da saúde é ainda tímida, segundo análise sistemática de iniciativas relatadas na literatura científica. Apesar deste panorama, a enfermagem é o campo que mais se destacou na produção destas ferramentas de ensino e aprendizagem, bem como avaliação das mesmas ${ }^{(1)}$.

As vantagens do uso de ferramentas tecnológicas que justificam sua utilização consistem em oferecer ao usuário fácil compreensão do assunto abordado(2), respeitar o ritmo de aprendizagem e possibilitar tanto a reutilização como facilidade de navegação entre os conteúdos $^{(1,3-4)}$, criar um ambiente seguro e eficaz para simular a prática clínica quando dotada de recursos mais avançados $^{(5)}$, desenvolver interesse devido suas peculiaridades gráficas $^{(4)}$ e promover satisfação dos usuários $^{(5)}$. Além disso, o uso dos ODA embasado pelas metodologias ativas estimulam os estudantes à colaboração com uso de meios eletrônicos. Desta forma, as distâncias geográficas deixam de ser empecilho para o trabalho em grupo que passa a ser incorporado no ensino a distância, desde que sejam oferecidos os recursos multimídia necessários e que as tarefas sejam alinhadas com essa proposta ${ }^{(6)}$. Entretanto, é fundamental refletir acerca das desvantagens a fim de melhor planejar o desenvolvimento e implementação dessas ferramentas, destacando: custo elevado ${ }^{(7)}$, risco de isolamento social na aprendizagem ${ }^{(7)}$ e subutilização do recurso justificada por desinteresse ou insatisfação ${ }^{(8)}$.

Considerando a hipótese de que o ODA de avaliação clínica do prematuro auxilia na aprendizagem cognitiva dos estudantes e favorece a motivação para os estudos no tema, a justificativa deste trabalho é fundamentada na preocupação em investigar o real impacto da ferramenta no desempenho dos estudantes em avaliação de aprendizagem e a opinião destes sobre seu uso.

Os objetivos deste trabalho foram: 1. Comparar o desempenho na aprendizagem dos estudantes pertencentes ao grupo controle em relação ao grupo experimental, adotando como intervenção o uso do ODA sobre avaliação clínica do prematuro e 2. Avaliar o ODA sob a ótica dos estudantes que o utilizaram (grupo experimental).

\section{MÉTODO}

Estudo quase-experimental realizado junto a estudantes de graduação de enfermagem de uma universidade pública do interior do sudeste brasileiro, que foram divididos entre grupos controle e experimental e avaliados por meio de pré e pós-teste.

Os estudantes foram convidados a participar do estudo por pesquisador desconhecido pelos estudantes, de modo a garantir que não houvesse conflito de interesses na relação professor-aluno. Mediante leitura do Termo de Consentimento Livre e Esclarecido, compreensão e concordância com o documento, os estudantes assinaram e dataram o mesmo em duas vias procedendo conforme a legislação brasileira vigente de ética em pesquisa. Esta pesquisa foi aprovada pelo Comitê de Ética em Pesquisa da Escola de Enfermagem de Ribeirão Preto da Universidade de São Paulo sob o protocolo $1220 / 2010$.

O convite de participação foi realizado a todos os 22 alunos matriculados na disciplina, pois atendiam aos critérios de inclusão adotados: 1. Estar matriculado na disciplina de saúde da criança (duração: 120 horas) e 2 . Participar de atividade prática de estágio em unidade neonatal prevista pelo curso. A organização da disciplina prevê a divisão prévia dos estudantes em dois grupos para viabilizar a execução de estágio hospitalar, sendo um grupo de 10 e outro de 12 estudantes. Desta forma, não houve alocação aleatória dos participantes nos grupos investigados, sendo o primeiro definido como controle $(n=10)$ e o segundo como experimental $(n=12)$. 
O ODA "Semiotécnica e Semiologia do Recémnascido Pré-termo" (SSRNPT) foi disponibilizado para acesso exclusivamente ao grupo experimental por meio do ambiente virtual de aprendizagem Moodle e controlado por meio de cadastro de login e senha.

O curso oferecido para os estudantes do grupo controle se diferenciou apenas quanto ao acesso da ferramenta adotada neste estudo enquanto intervenção, uma vez que as oportunidades de aprendizagem e o método pedagógico empregado foram os mesmos para ambos os grupos como já é naturalmente realizado na universidade.
Os recursos utilizados no ODA são compostos por vídeos, fotos, animações, figuras, sons, textos e questões sobre a avaliação clínica do prematuro. Em se tratando de uma ferramenta computacional com navegação livre oferece maior autonomia ao usuário, tendo este a oportunidade de estudar o que deseja, quando e onde quiser, sem necessitar completar módulos préestabelecidos para avançar nos conteúdos. A Figura 1 abaixo, apresenta a tela inicial do ODA Semiologia e Semiotécnica do recém-nascido pré-termo:

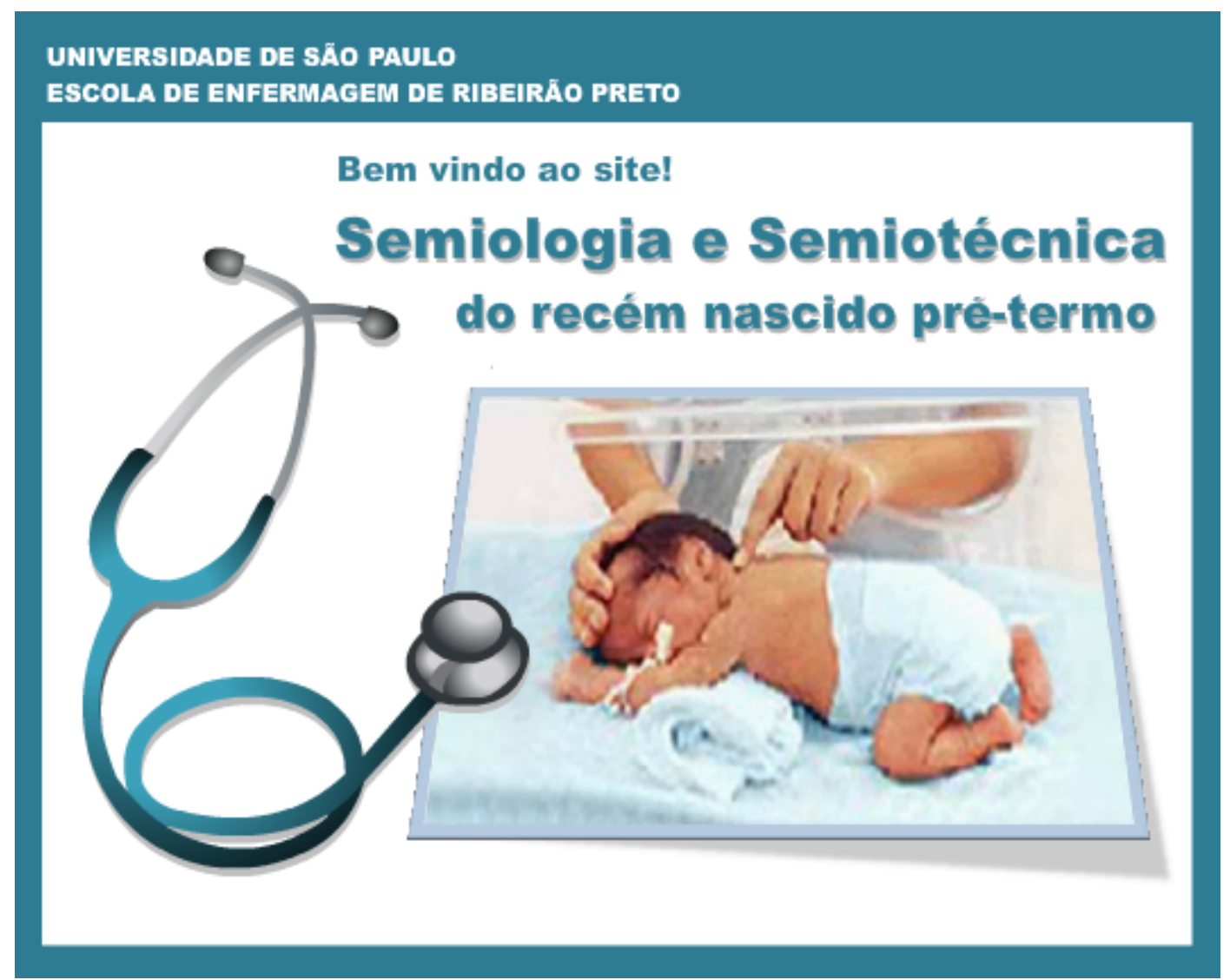

Figura 1: Tela de abertura do ODA Semiologia e semiotécnica do recém-nascido pré-termo. Ribeirão Preto, SP, Brasil, 2011.

Os instrumentos utilizados para a coleta de dados foram: Formulário de caracterização dos participantes; Questionário de pré e pós-teste composto por 20 questões objetivas incluindo animações multimídia e figuras e, Questionário de avaliação da opinião dos estudantes sobre o ODA (para aqueles que utilizaram a ferramenta) contendo 12 itens com base em escala Likert.
Para a avaliação da aprendizagem cognitiva, o desempenho dos estudantes foi considerado como sendo a diferença entre os escores de pós-teste e pré-teste (valor subtraído nesta ordem, possibilitando inclusive a obtenção de desempenho negativo - quando o estudante se saísse melhor no pré-teste).

Os testes estatísticos foram realizados por meio do programa SPSS (Statistical Package for the Social 
Sciences) utilizando método de análise não-paramétrica: Mann-Whitney na comparação entre os grupos e Wilcoxon para avaliar o desempenho antes e após a disciplina com e sem a intervenção dentro de um mesmo grupo. Todos os testes consideraram nível de significância igual a 0,05 .

\section{RESULTADOS}

Diante dos resultados encontrados neste estudo, as variáveis descritivas de caracterização dos participantes são apresentadas na Tabela 1.

Tabela 1: Variáveis descritivas para caracterização dos grupos controle e experimental. Ribeirão Preto, SP, Brasil, 2011

\begin{tabular}{|c|c|c|c|c|}
\hline \multirow{2}{*}{ Variável } & \multicolumn{2}{|c|}{ Controle } & \multicolumn{2}{|c|}{ Experimental } \\
\hline & $\mathbf{n}$ & (\%) & $\mathbf{n}$ & (\%) \\
\hline \multicolumn{5}{|l|}{ Frequência* } \\
\hline Frequentemente & 7 & 70 & 10 & 100 \\
\hline Regularmente & 2 & 20 & 0 & 0 \\
\hline Às vezes & 1 & 10 & 0 & 0 \\
\hline Nunca & 0 & 0 & 0 & 0 \\
\hline \multicolumn{5}{|l|}{ Sexo } \\
\hline Masculino & 4 & 40 & 1 & 8,3 \\
\hline Feminino & 6 & 60 & 11 & 91,7 \\
\hline \multicolumn{5}{|l|}{ Acessa o computador em casa } \\
\hline Não & 2 & 20 & 2 & 16,7 \\
\hline $\operatorname{Sim}$ & 8 & 80 & 10 & 83,3 \\
\hline \multicolumn{5}{|l|}{ Acessa o computador na universidade } \\
\hline Não & 0 & 0 & 1 & 8,3 \\
\hline Sim & 10 & 100 & 11 & 91,7 \\
\hline \multicolumn{5}{|l|}{ Acessa o computador no trabalho } \\
\hline Não & 9 & 90 & 12 & 100 \\
\hline Sim & 1 & 10 & 0 & 0 \\
\hline \multicolumn{5}{|l|}{ Acessa o computador em outro lugar } \\
\hline Não & 10 & 100 & 11 & 91,7 \\
\hline Sim & 0 & 0 & 1 & 8,3 \\
\hline \multicolumn{5}{|c|}{ Já pesquisou informações referentes ao curso na internet } \\
\hline Não & 0 & 0 & 0 & 0 \\
\hline Sim & 10 & 100 & 12 & 100 \\
\hline \multicolumn{5}{|c|}{ Já pesquisou avaliação clínica ou prematuridade na internet** } \\
\hline Não & 0 & 0 & 2 & 18,2 \\
\hline Sim & 10 & 100 & 9 & 81,8 \\
\hline \multicolumn{5}{|l|}{ Trabalha } \\
\hline Não & 8 & 80 & 10 & 83,3 \\
\hline Sim & 2 & 20 & 2 & 16,7 \\
\hline
\end{tabular}

Dos dados acima apresentados, destacamos os locais onde os estudantes mais utilizam o computador: casa ( $n=8 / 80 \%$ no grupo controle e $n=10 / 83,3 \%$ no grupo experimental) e universidade $(n=10 / 100 \%$ no grupo controle e $n=11 / 91,7 \%$ no grupo experimental).

Outro aspecto que deve ser ressaltado ao se observar a Tabela 1 se refere à grande quantidade de estudantes que já pesquisaram na internet a respeito de avaliação clínica ou prematuridade. Isto demonstra que já se interessaram por conhecer e estudar conteúdos relacionados ao tema, durante as demais disciplinas oferecidas no curso de enfermagem.

A média de acesso à internet, em horas, demonstrou que os participantes alocados no grupo experimental utilizam mais a internet $(15,17$ horas por semana) em relação ao controle $(9,11$ horas semanais) aumentando a possibilidade de acesso ao ambiente virtual de 
aprendizagem pelos estudantes que tiveram acesso liberado ao ODA neste estudo.

A comparação entre os grupos no que tange a aprendizagem cognitiva com base no desempenho segue representada pela Figura 2. O teste de Mann-Whitney demonstrou não haver diferença significativa entre os grupos investigados $(p=0,127)$.
Com relação ao desempenho dos estudantes ao se comparar os escores do pós-teste em relação ao pré-teste dentro de um mesmo grupo, o teste de Wilcoxon evidenciou melhora significativa nos dois grupos, considerados um a um, tanto o controle $(p=0,008)$ quanto o experimental $(p=0,025)$, conforme as Figuras 3 e 4 .

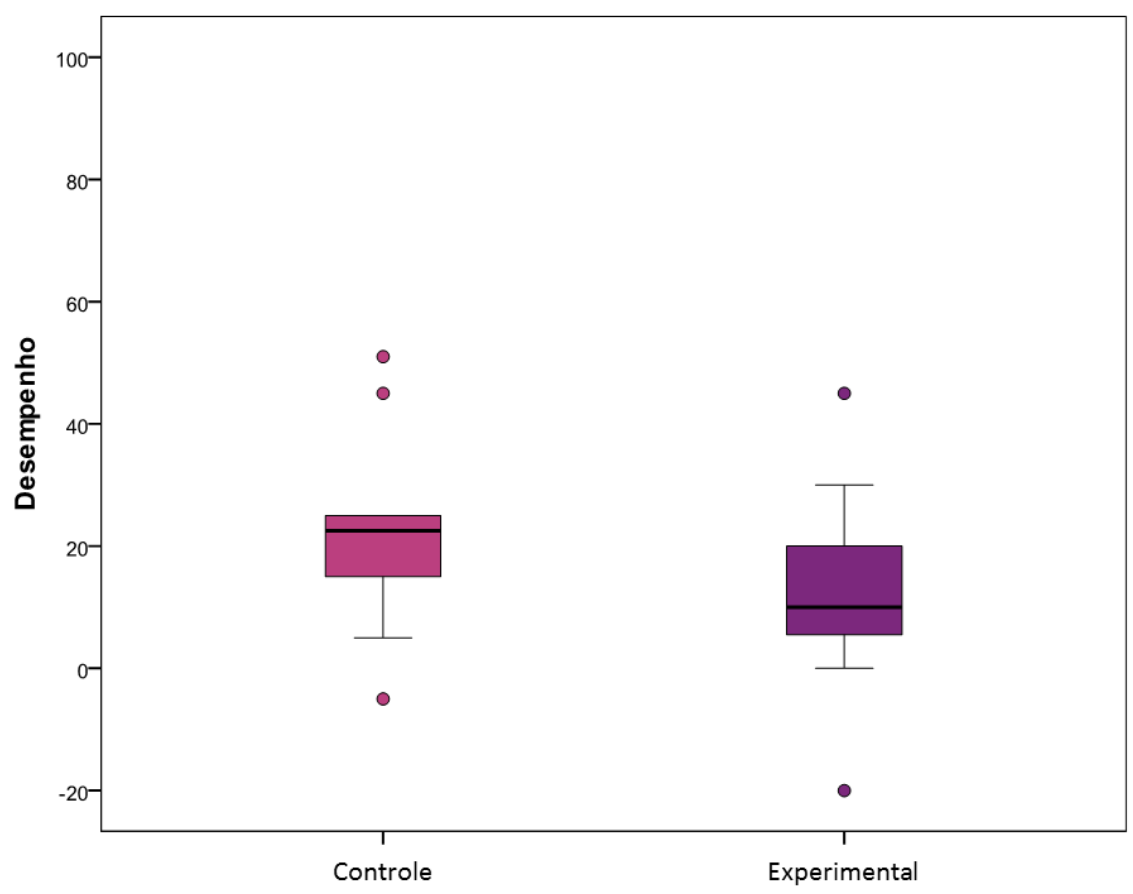

Figura 2: Comparação do desempenho entre os grupos controle e experimental sobre a aprendizagem cognitiva do exame clínico do recém-nascido prematuro. Ribeirão Preto, SP, Brasil 2011.

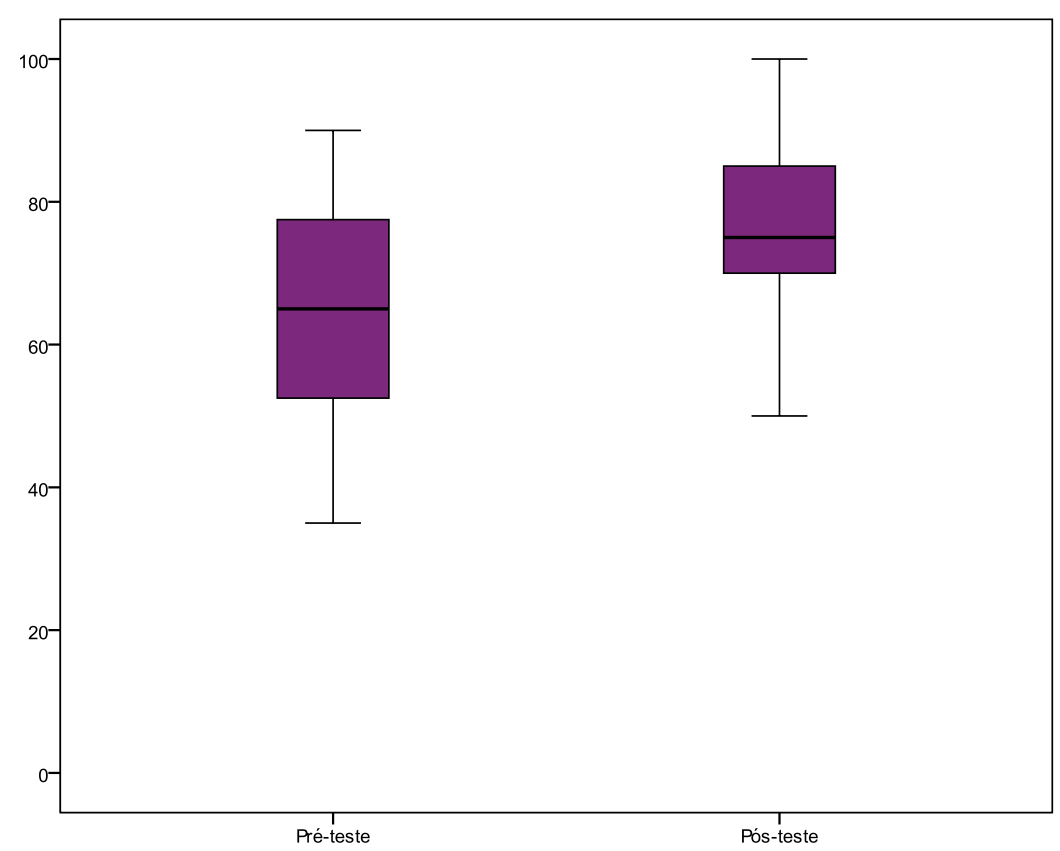

Figura 3: Desempenho dos estudantes do grupo experimental comparando aprendizagem cognitiva no pré e pós-testes. Ribeirão Preto, SP, Brasil, 2011. 


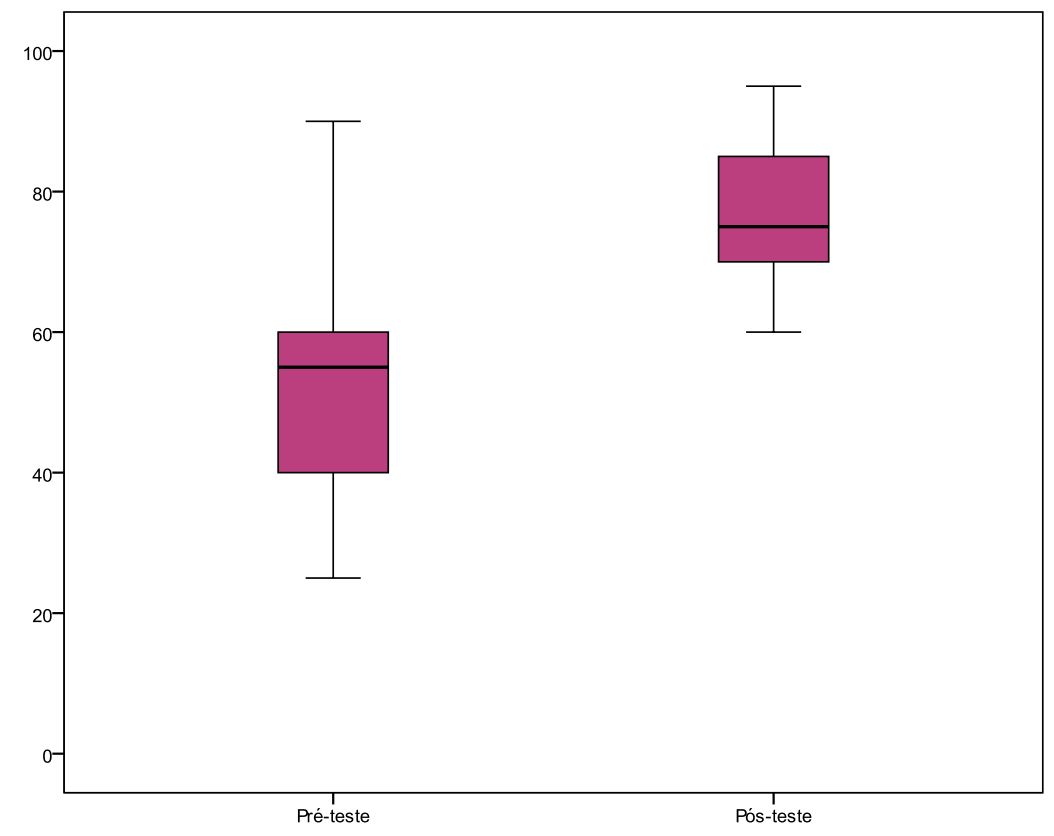

Figura 4: Desempenho dos estudantes do grupo controle comparando aprendizagem cognitiva no pré e pós-testes. Ribeirão Preto, SP, Brasil, 2011.

Na análise do instrumento de avaliação de opinião dos possibilidade de substituição dos professores por estudantes sobre o ODA, a expressiva maioria concorda recursos tecnológicos como o ODA (Tabela 2).

que a tecnologia educacional é satisfatória e refuta a

Tabela 2: Frequências de respostas sobre a avaliação subjetiva do ODA por estudantes do grupo experimental. Ribeirão Preto, SP, Brasil, 2011.

\begin{tabular}{|c|c|c|c|c|c|c|c|c|c|c|}
\hline \multirow{2}{*}{ Característica do ODA } & \multicolumn{2}{|c|}{ CP } & \multicolumn{2}{|c|}{ C } & \multicolumn{2}{|c|}{ NCND } & \multicolumn{2}{|c|}{ D } & \multicolumn{2}{|c|}{ DP } \\
\hline & $n$ & $(\%)$ & $\mathbf{n}$ & $(\%)$ & $\mathbf{n}$ & (\%) & $\mathbf{n}$ & (\%) & $\mathbf{n}$ & (\%) \\
\hline Fácil de usar & 8 & 66,7 & 4 & 33,3 & 0 & 0 & 0 & 0 & 0 & 0 \\
\hline Agradável & 8 & 66,7 & 4 & 33,3 & 0 & 0 & 0 & 0 & 0 & 0 \\
\hline Proporciona feedback imediato & 9 & 75 & 3 & 25 & 0 & 0 & 0 & 0 & 0 & 0 \\
\hline Didático & 10 & 83,3 & 2 & 16,7 & 0 & 0 & 0 & 0 & 0 & 0 \\
\hline Proporciona autonomia & 10 & 83,3 & 1 & 8,3 & 1 & 8,3 & 0 & 0 & 0 & 0 \\
\hline $\begin{array}{l}\text { Possibilita aprender as necessidades humanas básicas do } \\
\text { prematuro }\end{array}$ & 8 & 66,7 & 4 & 33,3 & 0 & 0 & 0 & 0 & 0 & 0 \\
\hline Posso escolher o que quero aprender & 5 & 41,7 & 7 & 58,3 & 0 & 0 & 0 & 0 & 0 & 0 \\
\hline $\begin{array}{l}\text { Seria interessante ter objetos digitais como este com outros } \\
\text { temas }\end{array}$ & 10 & 83,3 & 2 & 16,7 & 0 & 0 & 0 & 0 & 0 & 0 \\
\hline Objetos digitais assim podem substituir o professor & 1 & 8,3 & 0 & 0 & 2 & 16,7 & 5 & 41,7 & 4 & 33,3 \\
\hline O uso do objeto digital ajudou no meu aprendizado & 10 & 83,3 & 2 & 16,7 & 0 & 0 & 0 & 0 & 0 & 0 \\
\hline Eu me senti motivado a utilizar o objeto digital & 6 & 50 & 6 & 50 & 0 & 0 & 0 & 0 & 0 & 0 \\
\hline $\begin{array}{l}\text { Eu acredito que o tempo de acesso ao objeto digital foi } \\
\text { satisfatório para enriquecer meu aprendizado }\end{array}$ & 5 & 41,7 & 6 & 50 & 0 & 0 & 1 & 8,3 & 0 & 0 \\
\hline
\end{tabular}

$\mathrm{CP}=$ Concordo plenamente; $\mathrm{C}=$ Concordo; $\mathrm{NCND}=$ Não concordo nem discordo; $\mathrm{D}=$ Discordo; $\mathrm{DP}=$ Discordo plenamente

Destacamos alguns comentários dos estudantes que auxiliam na compreensão dos resultados obtidos neste estudo.

Embora a facilidade tenha sido referida de forma unânime, um estudante complementou: ... mas requer prática e habilidade para o estudo (Aluno C).
Sobre a liberdade de escolha do que aprender, todos os estudantes concordaram que o ODA oferece tal vantagem: Creio que o ODA ajudou no aprendizado e que podemos escolher o que estudar (Aluno C).

Todos os estudantes julgaram que o ODA apresenta feedback imediato: foi uma ferramenta interessante de 
aprendizagem, principalmente por ser possível visualizar no ato a resposta correta (Aluno F).

É possível identificar que os estudantes majoritariamente (75\%) "concordam totalmente" que o ODA é didático, sendo que os demais também "concordam" com esta afirmação. Um estudante afirmou que: foi uma ferramenta interessante de aprendizagem (Aluno D).

Outro aspecto importante que retratou o interesse dos estudantes nas ferramentas educacionais digitais foi a sugestão de continuidade no desenvolvimento de recursos como o utilizado neste estudo: e seja expandido para outras disciplinas e temas (Aluno B) e muito bom, este poderia ser expandido para outras disciplinas (Aluno E).

\section{DISCUSSÃO}

Reconhecendo a pluralidade nas formas de estudar e aprender, acreditamos que o professor deva buscar o equilíbrio entre as necessidades, expectativas e perfis de aprendizagem dos estudantes no ensino, bem como demandar estratégias diferenciadas e que propiciem aos alunos ter papel ativo e participativo em seu processo de ensino-aprendizagem. Dentre essas estratégias, enfatizamos a implementação da inovação tecnológica para a educação por facilitar a compreensão de informações complexas e atender as preferências dos jovens.

Pesquisa $^{(9)}$ que ressalta a diferença dos perfis de aprendizagem dos estudantes e discorre sobre a aceitabilidade da informática na rotina de aprendizagem, aponta aspectos que necessitam ser considerados no contexto das universidades e levados em conta ao se definir as estratégias de ensino. Mesmo considerando a individualidade de cada estudante, as turmas ou grupos possuem características que os unificam ao se considerar questões como idade semelhante, cultura e valores de uma geração ${ }^{(10-11)}$. Os resultados do presente estudo corroboram esta afirmação quando se observa a homogeneidade nas respostas altamente positivas quanto à avaliação de opinião sobre o ODA e convidam os docentes a refletirem sobre o papel das ferramentas inovadoras nas salas de aula e além do próprio espaço da universidade, também em um espaço digital viabilizado pela internet.

Os universitários de hoje fazem parte de uma nova geração, denominada por alguns como "nativos digitais" que parecem apresentar necessidades de aprendizagem muito diferentes do que as gerações passadas manifestaram. Estudos afirmam que os jovens da atualidade aprendem mais facilmente quando $o$ aprendizado se relaciona com a tecnologia e se inclinam positivamente para a realização de tarefas em grupo, baseadas em experiências, problemas e aprendizado colaborativo e interativo(12) ${ }^{(12)}$ Embora os resultados do estudo não tenham apresentado diferença estatisticamente significativa quando a ferramenta digital foi aplicada no ensino de enfermagem, à semelhança de outros estudos já realizados ${ }^{(7,12)}$, destacamos a satisfação dos estudantes que fortalecem os achados das pesquisas supracitadas.

Uma pesquisa realizada com 117 estudantes avaliou a aprendizagem em três grupos: ODA e leitura $(n=37)$, ODA, leitura e demonstração $(n=40)$ e leitura e demonstração $(n=40)$ e, semelhantemente aos resultados obtidos neste estudo, também não houve diferença significativa entre os grupos, porém foi demonstrado que o auxílio do objeto digital potencializa o desenvolvimento de habilidades ${ }^{(8)}$. Por outro lado, um estudo anterior que comparou dois grupos de enfermeiros atuantes na prática clínica quanto aos conhecimentos cognitivos sobre injeção intravenosa verificaram diferença estatisticamente significativa com vantagem ao grupo que utilizou o recurso multimídia ${ }^{(13)}$. Analisando a literatura, identificamos necessidade de mais investigações sobre o impacto do ODA na aprendizagem com estudos de maior rigor incluindo maior amostra e cálculo amostral, randomização dos participantes na alocação entre os grupos e controle de variáveis de confusão. 
Em relação à comparação intragrupos, outro estudo realizado no Brasil neste molde com estudantes de enfermagem $^{(12)}$ também obteve resultado de diferença significativa nas médias dos alunos no pré e pós teste à semelhança da presente investigação. Houve aumento importante na média considerada parâmetro para medir o desempenho dos estudantes, com base na aprendizagem cognitiva e os autores atribuíram parte do sucesso ao uso da tecnologia educacional.

Embora alguns estudos demonstrem não haver diferença significativa do ponto de vista estatístico, em geral os grupos experimentais que utilizam a tecnologia como ferramenta auxiliar no processo educacional se sobressaem em média no que tange avaliação cognitiva $^{(14)}$ ou satisfação sob o ponto de vista dos usuários $^{(15-16)}$, o que suscita reflexão acerca da análise de importância desses objetos digitais. É importante para o sucesso do uso das ferramentas educacionais que o contexto pedagógico o favoreça. Cabe ao docente, então, reconhecer as necessidades de aprendizagem e os momentos do processo educativo para lançar mão de estratégias e materiais de apoio, assim como utilizar os recursos disponíveis.

Neste estudo, os estudantes manifestaram alto nível de satisfação com o uso do ODA na disciplina de saúde da criança e todos concordaram com a facilidade do manuseio. Tal aspecto deve ser considerado na implementação de recursos tecnológicos na educação por viabilizar e estimular seu uso pelos estudantes ${ }^{(3,17)}$, ou seja, a facilidade do uso e a interação entre usuário e ODA devem existir na interface enquanto fatores críticos de sucesso. O reconhecimento das principais dificuldades dos estudantes acerca do uso de recursos tecnológicos em ambientes virtuais é muito importante, pois por esta estratégia de mapeamento é possível propor soluções para melhor aproveitamento dos mesmos ${ }^{(8)}$.

A dinamicidade e design dos recursos digitais estão diretamente relacionados ao bom aproveitamento das ferramentas, uma vez que além da qualidade do conteúdo, a aparência motiva o acesso e manuseio, torna a navegação agradável e estimula a busca por novos conhecimentos. Esta relação está atrelada ao conceito do design emocional ${ }^{(18)}$, que constitui uma área nova de estudo e desenvolvimento de produtos que visam contribuir na interação entre estes e seus consumidores. Trata-se de um termo utilizado em áreas do conhecimento como o marketing, por exemplo, e neste caso, por se tratar de educação, o design emocional pretende contribuir com o desenvolvimento de ferramentas educacionais capazes de despertar no usuário criatividade, curiosidade e motivação para o estudo com base na emoção positiva frente ao recurso mediador da educação.

No que se refere ao ODA ser agradável de usar, verificamos unanimidade em concordância pelos participantes assim como ocorreu em um estudo realizado sobre um ODA de verificação de sinais vitais ${ }^{(3)}$. A usabilidade está intimamente ligada à satisfação do usuário e deve ser considerada para o alinhamento das expectativas dos estudantes frente às tecnologias educacionais.

O feedback imediato tem grande responsabilidade sobre o sucesso de um ODA, uma vez que pode sanar, por meio da resposta rápida, dúvidas dos usuários quanto a um determinado assunto no momento em que elas surgem. Por ser estratégia da educação problematizadora, o feedback imediato aumenta a autonomia do estudante em auto-regular sua própria aprendizagem, podendo a partir dele desconstruir e reconstruir conceitos sobre determinado conteúdo(19). Este mecanismo faz parte do processo de construção de raciocínio crítico sobre os temas abordados e favorece as discussões subsequentes, bem como amplia o olhar do graduando ao lidar com uma situação similar na prática.

Os estudantes avaliaram muito positivamente a possibilidade de aprender o tema proposto com o ODA, satisfazendo a proposta de criação da tecnologia educacional reutilizável além de ser um fator motivador ${ }^{(4)}$ manifestado pelo interesse de expandir a iniciativa digital 
para outros temas, de acordo com os resultados obtidos na avaliação dos estudantes na presente investigação.

Quanto ao item que sugere que a figura do professor pode ser substituída pelos ODA, verificamos uma expressiva discordância dos participantes explicitando a relação que as tecnologias educacionais devem ter, ou seja, de ferramenta auxiliar e não de ferramenta substitutiva do papel do docente, tampouco de solução isolada para os problemas da educação.

Os ODA devem ser utilizados enquanto suplemento no ensino, reforçando a ideia de instrumento que acrescenta, mas não exime a função do educador ${ }^{(17)}$. As tecnologias educacionais utilizadas como recursos didáticos na prática docente devem estimular o estudo e a busca constante por novos conhecimentos.

Todos os participantes do grupo experimental afirmaram concordar fortemente com o item "Eu me senti motivado a usar o objeto virtual", o que constitui aspecto fundamental, uma vez que se o aluno não tem desejo de acessá-lo haverá subutilização ${ }^{(4)}$. Satisfatoriamente, este estudo identificou que os participantes se sentiram motivados a fazer uso da ferramenta de ensino o que viabilizou os acessos durante a disciplina e encorajam o desenvolvimento e aplicação de estratégias como essa no ensino superior de enfermagem.

Apesar da motivação, é necessário considerar que falta de tempo em decorrência das muitas atividades do curso superior $^{(20)}$ pode diminuir o aproveitamento dos ODA pelos estudantes, sendo esta uma variável de interesse e que requer atenção dos docentes no planejamento curricular e suporte técnico.

Iniciativas com foco na motivação variam desde a adequada utilização dos recursos multimídia ${ }^{(4)}$, identificação das preferências dos estudantes frente às ferramentas tecnológicas e criação destas tendo como norteadores o design emocional e a interação humanocomputador $^{(21)}$. Temos trabalhado com base nestes aspectos no desenvolvimento de novos materiais de ensino e na investigação das preferências dos estudantes, bem como das tendências de implementação tecnológica no ensino que sejam pertinentes ao processo de ensinoaprendizagem, promovam motivação ao estudante e maiores possibilidades didáticas ao docente.

Quanto aos desafios do avanço de aplicação da tecnologia no ensino, as universidades ainda se deparam com um obstáculo básico: falta de fluência digital. Para superá-lo, são necessárias ações planejadas das universidades em possibilitar o acesso às tecnologias e promover a capacitação dos usuários, sendo eles docentes, funcionários atuantes nas instituições de ensino superior e estudantes ${ }^{(22)}$.

Embora existam limitações no estudo apresentado, como amostra reduzida e ausência no controle de acesso dos usuários, o mesmo contribuiu com os esclarecimentos acerca da utilização de tecnologias na graduação em enfermagem e demonstrou a importância da avaliação de objetos virtuais desenvolvidos e validados junto ao público para quem foi proposta a criação.

Acreditamos na importância de pesquisas científicas que investiguem o impacto das tecnologias de ensino, 0 padrão de adesão aos dispositivos desta nova geração de estudantes e as preferências dos mesmos. É preciso avançar nesta área de pesquisa para que possamos, em consonância com os interesses do governo brasileiro, progredir face às novas diretrizes do ensino superior para os cursos da saúde, especialmente. Neste contexto, consideramos que a implementação de tecnologias educacionais validadas possa contribuir com o caráter de ensino mais participativo, disponibilizando materiais para os estudantes utilizarem de acordo com as necessidades individuais e respeitando os diversos ritmos de aprendizagem.

\section{CONCLUSÃO}

A comparação entre os grupos de estudantes demonstrou que o uso do ODA SSRNPT não determinou aumento significativo na aprendizagem cognitiva dos participantes a partir da análise de desempenho. As contribuições do ODA estão relacionadas principalmente 
à satisfação dos usuários, conforme tabela apresentada nos resultados deste trabalho, propiciando ambiente de estudo agradável e que complementa outras atividades de ensino, como as aulas regularmente oferecidas no curso e a prática clínica do estudante em unidade neonatal. Deste modo, consiste em ferramenta de ensino interessante para incorporação no contexto educacional de enfermagem neonatal.

Recomendamos o desenvolvimento de outros estudos com ampliação da amostra e randomização dos indivíduos para verificação do impacto do uso do objeto digital de aprendizagem por meio de estatística paramétrica. Além disso, são necessários trabalhos que investiguem a associação entre o uso de ODA e a motivação dos estudantes em estudar. Tal importância se justifica pela necessidade de se valorizar a proatividade do estudante diante do seu próprio processo de aprendizagem nas universidades e interesse na formação de profissionais mais resolutivos, críticos e que busquem continuamente a melhoria de seus conhecimentos e ações.

\section{FINANCIAMENTO}

Este projeto de pesquisa recebeu apoio do CNPq (Conselho Nacional de Desenvolvimento Científico e Tecnológico) para sua realização.

10. Collins AS, Graves BA, Gullette D, Edwards R. Developing an interactive microsimulation method in pharmacology. Journal of Nursing Education, 2010; 49(7):410-3.

11. Gibson S. Enhancing intergenerational communication in the classroom: Recommendations for successful teacherstudent relationships. Nursing Education Perspectives, 2009; 30(1):37-9.

12. Alvarez AG, Dal Sasso GTM. Aplicação de objeto virtual de aprendizagem, para avaliação simulada de dor aguda, em estudantes de enfermagem. Revista Latino-Americana, 2011; 19(2):229-37.

13. Tsai SI, Tsai WW, Chai SK, Sung WH, Doong JL, Fung CP. Evaluation of computer-assisted instruction in intravenous injection. International Journal of Nursing Studies, 2004; 41(2):191-98.

14. Morey DJ. Development and Evaluation of Web-Based Animated Pedagogical Agents for Facilitating Critical Thinking in Nursing. Nursing Education Perspectives. 2013; 33(2):116120.

15. Anderson JK, Page AM, Wendorf DM. Avatar-Assisted Case Studies. Nurse Educator. 2013; 38(3):106-109.

16. Hessler KL, Henderson AM. Interactive Learning Research: Application of Cognitive Load Theory to Nursing Education. International Journal of Nursing Education Scholarship. 2013; 10(1):1-9.

17. Fonseca LMM, Aredes NDA, Chiodi LC, Furtado MCC, Leite AM, Martins JCA, Rodrigues MA. Serious game e-Baby e software de avaliação clínica do prematuro: influência na aprendizagem de estudantes de enfermagem no tema neonatal. In: IASIS - I workshop ibero-americano de sistemas interoperáveis em Saúde. Revista da Faculdade de Medicina e do Hospital das Clínicas de Ribeirão Preto. 2014; 47: 38-43. 18. Tonetto LM, Costa FCX. Design emocional: conceitos, abordagens e perspectivas de pesquisa. Strategic Design Research Journal, 2011; 4(3)132-140. 
19. Fonseca LMM, Aredes NDA, Dias DMV, Scochi CGS, Martins

JCA, Rodrigues MA. Serious game e-Baby: percepção dos

estudantes de enfermagem sobre a aprendizagem da avaliação clínica do bebê prematuro. Rev Bras Enferm, 2015; 68(1):13-

19.

20. Silva VLS, Chiquito NC, Andrade RAPO, Brito MFP, Camelo $\mathrm{SHH}$. Fatores de estresse no último ano do curso de graduação em enfermagem: percepção dos estudantes. Rev. enferm.

UERJ, 2011; 19(1):121-126.

21. Fonseca LMM, Dias DMV, Góes FSN, Seixas CA, Scochi CGS, Martins JCA, Rodrigues MA. Development of the e-Baby serious game with regard to the evaluation of oxygenation in preterm babies. Computers, Informatics, Nursing, 2014; 32(9):428-436. 22. NMC - New Media Consortium and The EDUCAUSE Learning Initiative. Johnson L, Adams Becker S, Estrada V, Freeman A. NMC Horizon Report: Higher Education Edition, 2014. ISBN 978-0-9897335-5-7. Austin, Texas: The New Media Consortium.

Recebido: 28/04/2015.

Aceito: 24/09/2015.

Publicado: 31/12/2015. 